\title{
Effects of Xylanase Pretreatment on the Quality of Refiner Mechanical Mulberry Branch Fibers
}

\author{
Wenjuan Tao, Lifang Guo (D), Aojie Meng, Lizhen Wang, Hao Ren (D), and Huamin Zhai (i) \\ Jiangsu Provincial Key Lab of Pulp and Paper Science and Technology, Nanjing Forestry University, China \\ Correspondence should be addressed to Huamin Zhai; hzhai@njfu.edu.cn
}

Received 31 March 2019; Revised 2 June 2019; Accepted 3 July 2019; Published 15 July 2019

Academic Editor: Sheldon Q. Shi

Copyright ( 2019 Wenjuan Tao et al. This is an open access article distributed under the Creative Commons Attribution License, which permits unrestricted use, distribution, and reproduction in any medium, provided the original work is properly cited.

\begin{abstract}
We performed xylanase pretreatment prior to mechanical refining in the production of mulberry branch fibers, with the objective of saving energy and studying the effects of such pretreatment on the quality of the fibers. To determine the effects of the enzyme action, we analyzed the energy required for refining, related yield, and the dimension, deformation, and morphology of the fibers. We found that, with the xylanase pretreatment, the refining energy was reduced by $4 \%$, with the yield of fibers being maintained at $>85 \%$. In addition, the fiber bundles were defibered further, resulting in reduced average length of the fiber. Furthermore, the fiber widths increased because of the improved swelling effect of the xylanase pretreatment. However, in some instances, the fine elements were reduced. With a low enzyme dosage, the fiber coarseness decreased remarkably and, because of the swelling and softening effects of the xylanase pretreatment on the mulberry branches, the fiber kink ratios and curl were reduced. Additionally, the mulberry branch tissue was loosened, facilitating fiber separation. In view of these findings, the biomechanical process could be a potentially green and efficient process for the manufacturing of mulberry branch fibers.
\end{abstract}

\section{Introduction}

As public awareness of the effects of economic growth and the attendant need for environmental protection increases, research interest in the utilization of renewable lignocellulosic biomass has increased $[1,2]$. One such biomass species is mulberry (Morus alba L), which belongs to the genus Morus of the family Moraceae [3] that is grown widely in China, Southern Europe, North America, East Asia, Southeast Asia, and Australia [4]. A mulberry branch comprises phloem $(\sim 27 \%)$, xylem $(\sim 72 \%)$, and pith $(\sim 1 \%)$, with a density of approximately $0.49 \mathrm{~kg} / \mathrm{m}^{3}$ [5]. Its main chemical components are cellulose, hemicellulose, lignin, pectin, and ash [6]. As mulberry branches are one of the by-products of the silkworm industry, using these branches as a fiber resource could represent significant added value.

The xylanolytic enzyme system comprises three synergistic biological enzymes, namely, endo- $\beta$-1,4-xylanase, exo- $\beta$-1,4-xylanase, and $\beta$-xylosidase [7], which are crucial enzymes in the hydrolysis of the $\beta$-1,4-xylosidic bond of the xylan polymer backbone. Xylanase has been used in various industrial processes, including the pulp and paper industry, textile processing, biofuel industry, organic waste treatment, and the food and feed industries [8]. In the treatment of lignocellulosic biomasses, xylanase is able not only to catalyze the hydrolysis of hemicellulose but also to remove an amount of lignin by degradation of the lignin-carbohydrate complex (LCC) [9]. In addition, xylanase treatment can be considered a modification method for lignocellulosic materials, as it can modify the physical and chemical structures of such materials by degradation of xylan and, thereby, can affect their performance in various applications [10-12].

Thermomechanical pulping (TMP) is a fiber refining process whereby pulp is generated by a high-temperature high-pressure steam treatment prior to mechanical refining. Although this process is well developed and produces high yields, its energy consumption is relatively high [13]. In addition to its application in the pulp industry, TMP has been used widely in studies involving wood-plastic composites and biomass conversion, among others [14-17]. Over the past few decades, significant research efforts have focused on optimizing the TMP process to reduce energy consumption, such as chemical pretreatment, extrusion before pulping, and hightemperature refining [18]. However, challenges related to the 
fiber quality and energy consumption remain. Therefore, improving the degree of fibrillation and the proportions of the long fibers is vital to improving the TMP technology [19]. As the fibrous tissue of wood is a complex biocomposite material, such issues will remain unsolved if the fibers were refined only by mechanical actions. Biopulping is the fungal or enzymatic pretreatment of wood chips for the production of mechanical or chemical pulps. It is an environmentally friendly technology that increases mill throughput substantially or reduces electrical energy consumption at the same throughput in conjunction with mechanical pulping [20]. In comparison with the widely studied degradation of wood fiber using white-rot fungi, enzymatic treatment appears to be a more rapid and efficient process [21]. Xylanase can diffuse into the interior of the fiber cell walls and act on both the fiber surface and the fiber interior [22]. The pretreatment of lignocellulosic biomass using heat- and alkali-resistant xylanase can activate and relax the fibers, which can enhance the swelling capacity and fibrillation of the fibers [23]. Consequently, the energy consumption required for pulping can be reduced and the pulp reaction performance can be improved [24-27]. As the mulberry fibers have a loose structure and high xylan content, xylanase pretreatment could be considered a promising solution to the problems mentioned [28, 29].

Fiber qualities are commonly characterized in terms of fiber length, width, coarseness, kinks, and curls. The fiber length and width are the most important indicators of fiber properties. Fiber coarseness is defined as the mass of fiber per unit length, and it is affected by the relative density, cell wall thickness, and the lumen size of the fiber. Fiber deformation is reflected by the curl and kinks in the fibers. These fiber morphology characteristics affect the softness, binding force, and water filtration performance of the fibers that, ultimately, affect the tearing strength, permeability, air permeability, and smoothness of any synthesized materials [18]. For example, when fibers are used in wood-plastic composites, fiber morphology plays a significant role in determining the properties of the composite materials [13]. Wood fibers can be subjected to physical, chemical, biological, or joint actions, which result in morphological changes that can further affect the structures and the mechanical and optical properties of the fiber products. Therefore, investigation of the effects of xylanase pretreatment on the morphological changes in mechanically refined mulberry fibers is of particular importance.

In this study, we report our investigation into the effects of xylanase pretreatment on the characteristics of refined mulberry branch fibers. More specifically, we investigate the effects of the xylanase pretreatment on the energy required for such mechanical refining, the fiber quality, and the fiber morphology in detail. The aim of the study is to determine how much energy can be saved in mechanical refining with xylanase pretreatment, as well as the effect of the enzyme pretreatment on the fiber dimension, coarseness, deformation, and morphology. We used various enzyme dosages to explore the full potential of xylanase pretreatment and we studied the changes in the fiber quality to determine the enzyme actions in the process. We expect that our findings will provide a theoretical basis for the green production of fibers from the entire stem of the mulberry branch using this combined biomechanical technique.

\section{Materials and Methods}

2.1. Materials. Mulberry (Morus alba L) tree branches were collected from Nantong City, Jiangsu Province, China. After the removal of impurities (sand, dust, and the like), the mulberry branches were cut into strips of 20-25 mm in length and 5-8 $\mathrm{mm}$ in width. The chemical components present in the mulberry branches are outlined in Table 1 .

The xylanase (Pulpzyme HC 2500) used in our experiment was provided by Novozymes (USA). The preferred operating conditions are a temperature range of $40-60^{\circ} \mathrm{C}$ and a $\mathrm{pH}$ range of 7-9.5. At $\mathrm{pH} 8.0$ (citric acid/sodium citrate buffer solution) and $50^{\circ} \mathrm{C}$, the enzyme activity of xylanase was $1631 \mathrm{U} / \mathrm{mL}$, without cellulase or laccase.

2.2. Xylanase/Disc-Refining Process for the Production of Mulberry Branch Fibers. The experimental procedure used in the study is outlined schematically in Figure 1.

2.2.1. Screw Extrusion. The wood chips were screened, immersed in tap water at approximately $25^{\circ} \mathrm{C}$ for $24 \mathrm{~h}$, and then subjected to spiral extrusion using a roll crusher (Andritz-Bauer 6" MSD press impregnator, Austria) at a compression ratio of 4:1. Following screw extrusion, the obtained materials were air dried at approximately $25^{\circ} \mathrm{C}$ prior to use.

2.2.2. Hot Water Pretreatment. The air-dried raw material (oven-dried mass $=200 \mathrm{~g}$ ) was placed in a plastic bag and sealed. Boiled deionized water was added at a solid/liquid ratio of 1:6 (wood:deionized water). After uniform mixing, the mixture was placed in a water bath at a constant temperature of $100^{\circ} \mathrm{C}$ for $30 \mathrm{~min}$.

2.2.3. Xylanase Pretreatment. After the thermal pretreatment, the mulberry branch samples (oven-dried mass $=200 \mathrm{~g}$ ) were placed in a plastic bag and sealed. Deionized water was subsequently added at a solid/liquid ratio of 1:10 (wood:deionized water). The $\mathrm{pH}$ was adjusted to approximately 9.0 using a solution of $0.1 \mathrm{~mol} / \mathrm{L} \mathrm{NaOH}$ and, subsequently, the desired quantity of the enzyme solution was added. The resulting substance was mixed uniformly and placed in a water bath at a constant temperature of $55^{\circ} \mathrm{C}$ for $60 \mathrm{~min}$. The bag was rubbed every $15 \mathrm{~min}$ to ensure uniform mixing. After this time, the mixture was dehydrated up to a dryness of $~ 30 \mathrm{wt} \%$ by using a centrifuge.

Our control samples were subjected to water pretreatment without the addition of xylanase. The remainder of the process was identical.

2.2.4. Presteaming. Presteaming was carried out in a highpressure steam sterilizer (Shanghai Boxun Industry \& Commerce Co., Ltd., China) at $125^{\circ} \mathrm{C}$ for $10 \mathrm{~min}$. 


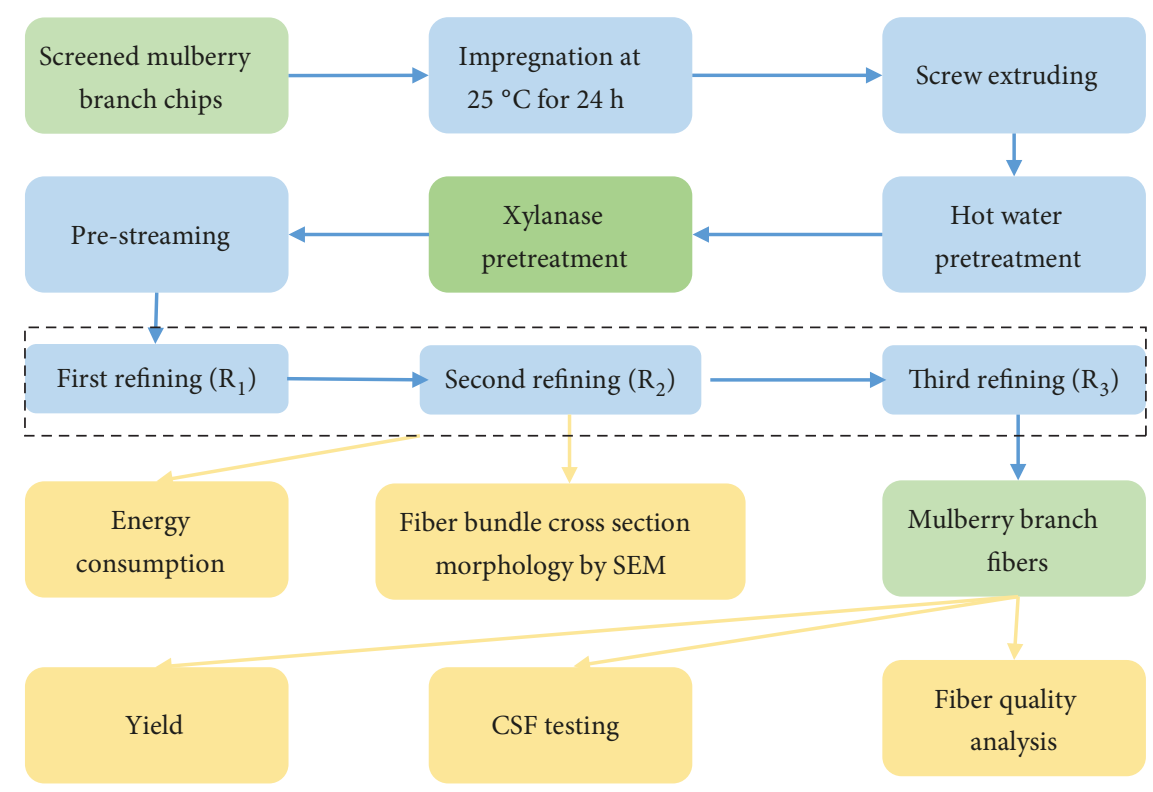

FIGURE 1: Experimental procedure.

2.2.5. Fiber Refining. The fiber refining process was carried out using a disc-refiner (RK85, Shaanxi Science and Technology Machinery Factory, China) at atmospheric pressure. The dry mass of each set of raw materials was $200 \mathrm{~g}$. The refining process was divided into three stages. In the first $\left(R_{1}\right)$ stage, the stock concentration was $25 \%$, and the disc gap setting was $0.25 \mathrm{~mm}$. In the second $\left(\mathrm{R}_{2}\right)$ stage, the concentration was $20 \%$, and the disc gap setting was $0.15 \mathrm{~mm}$, whereas in the third $\left(\mathrm{R}_{3}\right)$ stage, the concentration was $20 \%$, and the disc gap setting was $0.15 \mathrm{~mm}$.

2.3. Measurement of Energy Consumption. The precision of measuring the energy consumption during disc refining was $0.01 \mathrm{~kW} \cdot \mathrm{h}$. The specific energy consumed for pulping was calculated using the following equation:

$$
\begin{aligned}
& \text { Specific energy consumed for pulping }\left(\frac{\mathrm{kW} \cdot \mathrm{h}}{\mathrm{t}}\right) \\
& =\frac{\text { Total energy consumed for pulping }- \text { Energy consumed during idling }}{\text { Oven dry weight of pulp stock }}
\end{aligned}
$$

2.4. Freeness Measurements. The Canadian standard freeness (CSF) was measured using an appropriate tester (Canadian Standard Freeness Tester, P41510, PTI, Austria) according to the standard TAPPI T-227.

2.5. Fiber Quality Analysis (FQA). A sample, equivalent to an oven-dried stock weight of $40 \mathrm{mg}$, was weighed accurately, and the pulp was placed in a standard pulp disintegrator (PTI, Austria) for standardized disintegration to ensure that the interlaced fibers were freed in the pulp stock and were present as single fibers. Following pulp disintegration, the concentration was adjusted to $0.004 \mathrm{wt} \%$. The samples were analyzed using a fiber quality analyzer (FQA, Morfi, THCHPAP, France), and the distributions of the fiber fines and fiber mean length, as well as the mean kink and curl indices, were calculated based on data from approximately 5 000 fibers. Fiber fines were defined as the fiber portion with a length of 70-200 $\mu \mathrm{m}$, and the fiber length was determined over a range of $0.2-10.0 \mathrm{~mm}$. The aspect ratio was calculated by the ratio of the corresponding length and width. Two measurements were performed in parallel.

2.6. Scanning Electron Microscopy (SEM). Samples of the freeze-dried fiber were embedded, sliced, and mounted on the sample stage using conductive adhesive. Prior to morphological analysis, a layer of platinum-palladium $(\mathrm{Pt} / \mathrm{Pd})$ alloy, with a thickness of $3 \mathrm{~nm}$, was deposited on the surfaces of all the samples using ion sputter coating (Hitachi E1010, Japan). The sample morphology was examined using scanning electron microscopy (SEM, FEI Quanta 200, USA) at an accelerating voltage of $25.0 \mathrm{kV}$.

\section{Results and Discussions}

3.1. Effect of Xylanase Pretreatment on the Pulp Yield. Mulberry branches were treated with different xylanase dosages (i.e., $0,4,10$, and $20 \mathrm{U} / \mathrm{g}$ ), and the untreated sample (i.e., 
TABLE 1: Chemical components in the whole stem of mulberry branch.

\begin{tabular}{lcc}
\hline \multicolumn{2}{c}{ Components } & Content (\%) \\
\hline Extractions & & \\
& Benzene/alcohol & $1.98 \pm 0.02$ \\
& Hot water & $7.44 \pm 0.09$ \\
& $1 \% \mathrm{NaOH}$ & $29.3 \pm 0.2$ \\
Lignin & & \\
& Klason lignin & $23.5 \pm 0.2$ \\
& Acid-soluble lignin & $3.17 \pm 0.04$ \\
& Total & $26.7 \pm 0.2$ \\
Holocellulose & & $74.9 \pm 1.1$ \\
Hemicellulose & & $26.8 \pm 0.2$ \\
Ash & & $2.76 \pm 0.03$ \\
\hline
\end{tabular}

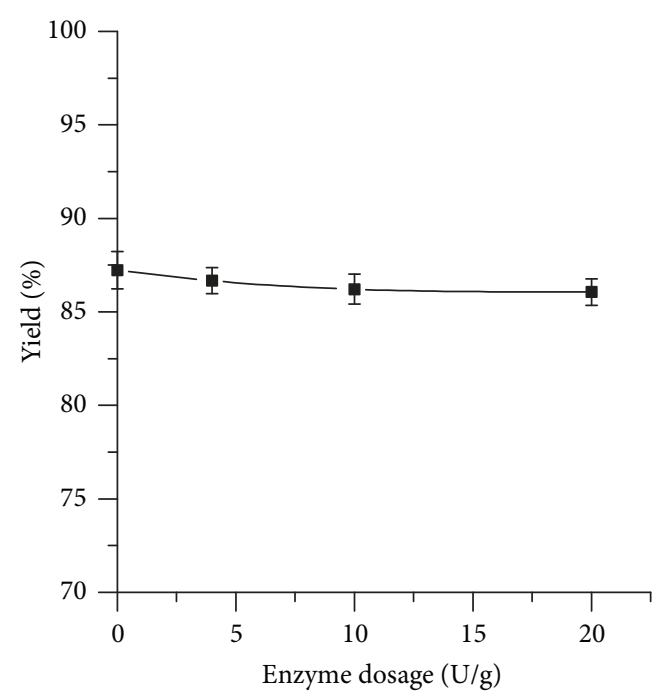

FIGURE 2: Effect of xylanase pretreatment on the yield of refiner mechanical fibers.

$0 \mathrm{U} / \mathrm{g}$ ) was used as the blank control. All other conditions were kept constant. The effect of the xylanase pretreatment on the pulp yield using whole stems of mulberry branches is shown in Figure 2. The figure indicates that increasing the enzyme dosage results in decreased pulp yield. When the enzyme dosage is $20 \mathrm{U} / \mathrm{g}$, the pulp yield is $86.0 \%$, which is a decrease of $1.3 \%$ compared with the control sample. The removal of xylan using xylanase results in loosened structures and increased porosity [30]. As the xylanase dosage is increased, the reaction between xylanase and xylan is promoted. The hemicellulose-carbohydrate complex (LCC) that is linked to the hemicellulose is degraded and detached by the xylanase actions. In addition, the dissolution of other components increases [24]. Therefore, all the factors result in lower yields. However, in our study, the raw materials used were mulberry branch slices with potentially limited accessible surface areas $[31,32]$, and, therefore, no significant decreases in yield were observed.

3.2. Effect of Xylanase Pretreatment on Energy Consumption. The dissociation of fibers by disc refining is divided generally into three steps, namely, crushing (the stock state changes from wood chips to matchstick-shaped small wood strips), rough grinding (the stock state changes from matchstickshaped small wood strips to needle-shaped wood wire, and then further to fiber bundles and some single fibers), and fine grinding (the fibrillation of fibers) [33, 34]. In the pulping process, energy is consumed mainly in the defibering and refining processes, with the refining stage consuming the most energy. The energy consumption of the refining stage is related to the swelling and softening state of the fibers $[34,35]$. The effect of the xylanase pretreatment on the energy required for the pulping of whole stems of mulberry branches is shown in Figure 3. As the enzyme dosage increases, the energy consumption decreases. For example, with an enzyme dosage of $4 \mathrm{U} / \mathrm{g}$, the energy consumption is reduced by $3 \%$ compared with the blank control sample. The change in energy consumption is less pronounced at higher enzyme dosages. The freeness of pulp is employed commonly to evaluate the degree of fiber dissociation [36]. As shown in Figure 2, the freeness increases by increasing the xylanase dosage. At an enzyme dosage of $4 \mathrm{U} / \mathrm{g}$, the freeness increases from $205 \mathrm{~mL}$ (the blank control sample) to $245 \mathrm{~mL}$. This change is less noticeable at higher enzyme dosages, similar to the finding on the change in energy consumption. In fiber cell walls, hemicellulose acts as the "binder" between cellulose and lignin. As the enzyme dosage is increased, the removal of hemicellulose increases, and the structures become loose, which is favorable to defibering. Consequently, the energy required for fiber refining is reduced [36]. However, the xylanase pretreatment increases the fiber freeness, which means an increase in fiber filtration capacity. This probably resulted from the degree of fiber dissociation, the content of fine fiber, and/or the degree of fibrillation. However, as indicated in Section 3.3 (changes in fiber quality and morphology) below, the degree of fiber dissociation does not decrease, whereas the fines content does decrease. Therefore, the reduction in energy consumption is probably related to the reduction in energy consumption in the refining stage after the xylanase treatment.

\subsection{Effect of Xylanase Pretreatment on Fiber Quality}

3.3.1. Fiber Length, Width, and Distribution. The effect of xylanase treatment on the fiber size is shown in Figure 4. With increasing xylanase dosage, the fiber length decreases, compared with the blank sample. At an enzyme dosage of $4 \mathrm{U} / \mathrm{g}$, the fiber length ( $0.44 \mathrm{~mm}$ in the control sample) is reduced to $0.43 \mathrm{~mm}$, i.e., by $2.3 \%$. At an enzyme dosage of 10 $\mathrm{U} / \mathrm{g}$, the fiber length is reduced to $0.40 \mathrm{~mm}$, i.e., a reduction of $9.1 \%$ compared with the control. At an enzyme dosage of $20 \mathrm{U} / \mathrm{g}$, the fiber length is $0.39 \mathrm{~mm}$, i.e., a decrease of $11.4 \%$ compared with the control. As shown in the fiber length distribution pattern (Figure 5), an increase in the enzyme dosage results in a significant decrease in the portion of fiber length measuring $0.20-0.29 \mathrm{~mm}$, whereas the portion measuring $0.29-0.84 \mathrm{~mm}$ increases significantly, and the proportion of fibers measuring $>0.84 \mathrm{~mm}$ declines. As regards fibers with a length of $>0.84 \mathrm{~mm}$, including relatively long fibers and fiber bundles, the higher the enzyme dosage is the 


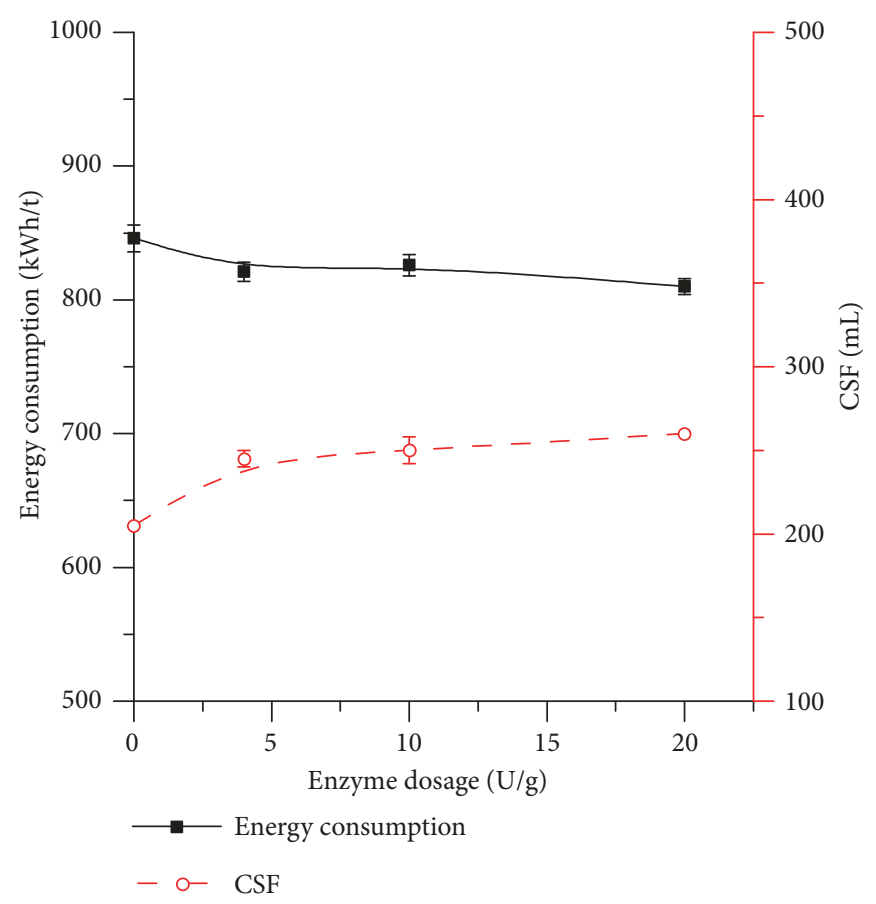

FIGURE 3: Effect of xylanase pretreatment on the mechanical refining of mulberry branches and the required energy consumption.

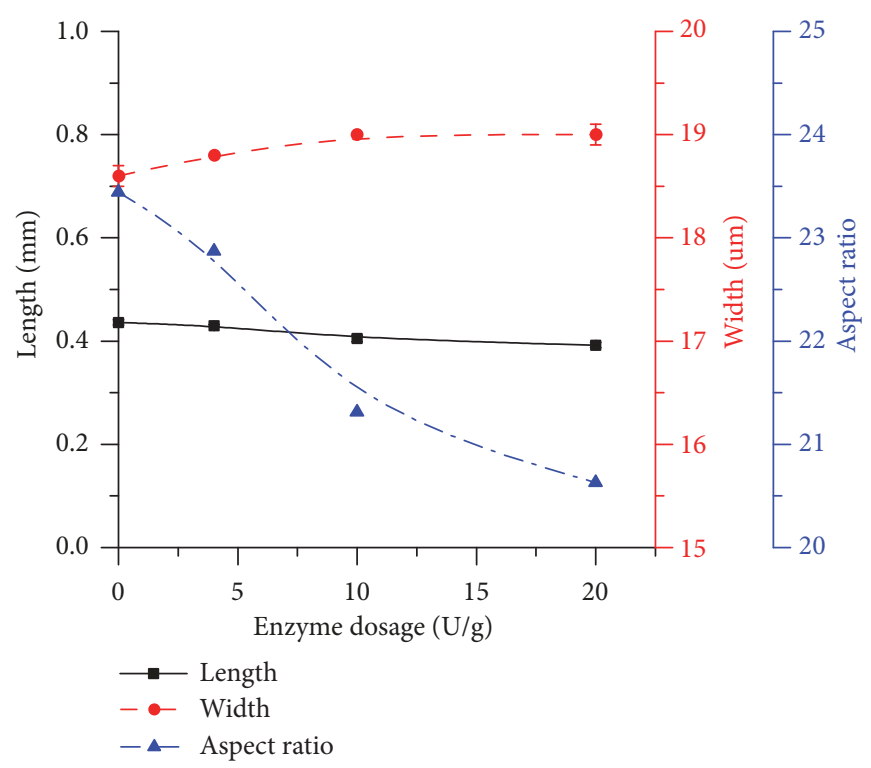

FIGURE 4: Effect of xylanase treatment on the length and width of refiner mechanical fibers.

lower would be the proportion of such fibers. This indicates that the xylanase treatment was beneficial to defibering and the higher the dosage is, the better would be the effect. As regards the portions of fiber measuring $0.41-0.84 \mathrm{~mm}$, including single long fibers and fiber bundles, the proportion in the xylanase-treated sample increases to a certain extent compared with the control. However, it decreases with an increase in the enzyme dosage. This can be ascribed probably to the two types of changes occurring simultaneously with the xylanase pretreatment. The defibration of the longer fibers $(>0.84 \mathrm{~mm})$ leads to an increase in the proportion of shorter fibers $(0.41-0.84 \mathrm{~mm})$ and the xylanase treatment contributes to defibering this portion even further. When the enzyme dosage is higher, more bundles are defibered into single fibers, which results in a significant increase in the proportion measuring $0.29-0.41 \mathrm{~mm}$. As regards the portion of fibers measuring $0.29-0.41 \mathrm{~mm}$, i.e., almost single fibers, this proportion increases with the increasing enzyme dosage. However, as regards the portion measuring $0.20-0.29 \mathrm{~mm}$, i.e., shorter fibers and broken fiber fragments, this proportion decreases. These results suggested that the reduction in fiber length could be ascribed to a decrease in 


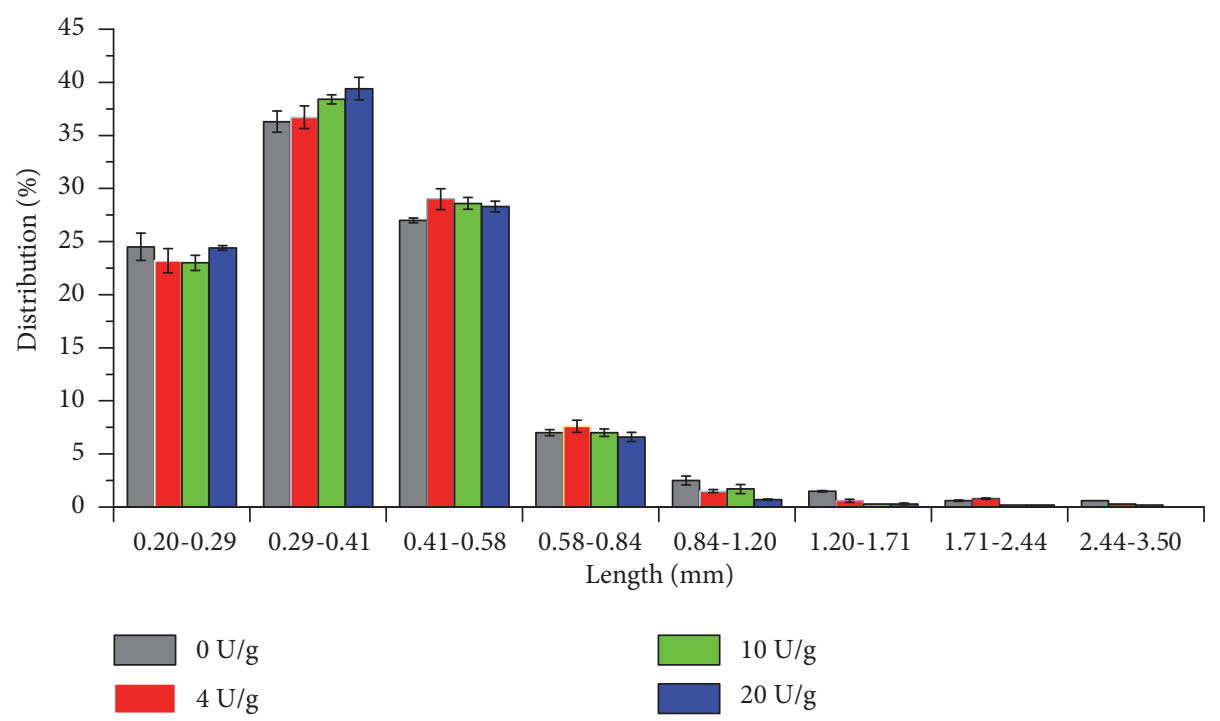

FIGURE 5: Effect of xylanase treatment on the length distribution of refiner mechanical fibers.

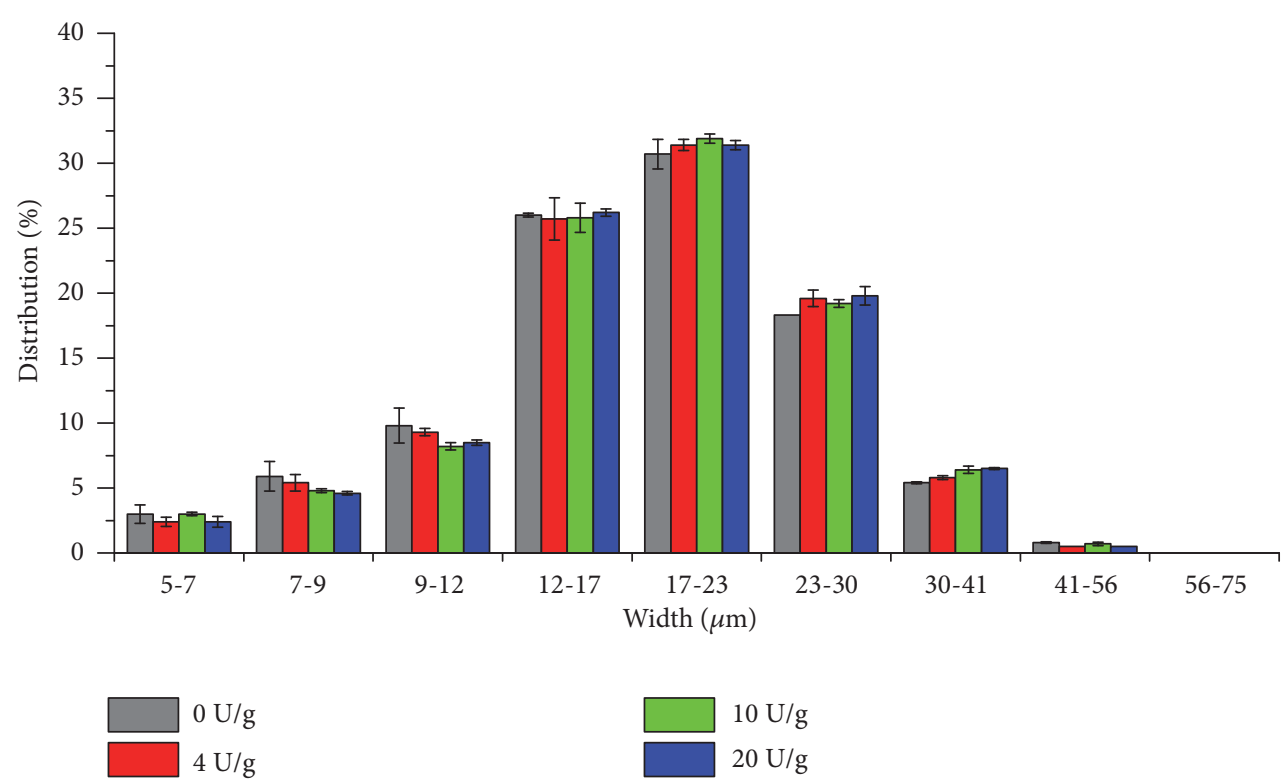

FIGURE 6: Effect of xylanase treatment on the width distribution of refiner mechanical fibers.

the number of fiber bundles and not to the cutting of the fibers.

The effects of the xylanase pretreatment on the average fiber widths are shown in Figure 4. With an increase in the enzyme dosage from 0 to $10 \mathrm{U} / \mathrm{g}$, the average fiber width increases from $18.6 \mu \mathrm{m}$ (the blank control sample) to 19.0 $\mu \mathrm{m}$ (i.e., an increase of $2.2 \%$ ). When the enzyme dosage increases from 10 to $20 \mathrm{U} / \mathrm{g}$, no significant changes in the fiber width are observed. Therefore, as shown in the fiber width distribution pattern presented in Figure 6, an increase in the enzyme dosage results in a decrease in the percentage of fiber with a width range of 5-17 $\mu \mathrm{m}$ (mainly the single fiber width range). This is attributed to the xylanase pretreatment improving the water absorption and swelling capability of the fibers. However, the percentage of the fiber width in the range $41-56 \mu \mathrm{m}$ (i.e., the fiber bundle range) decreases, which further confirms that the enzymatic treatment facilitates the dissociation of fiber bundles. In addition, the corresponding aspect ratio decreases with the increase in the enzyme dosage because of the change in fiber length and width.

3.3.2. Fiber Coarseness. The effect of the xylanase treatment on the coarseness of the refiner mechanical fibers is shown in Figure 7. An increase in the xylanase dosage results in an initial decrease in the coarseness of the fibers, followed by a subsequent increase. When the enzyme dosage increases from 0 to $4 \mathrm{U} / \mathrm{g}$, the fiber coarseness decreases significantly. As previously discussed in relation to Figure 3, no significant changes in the fiber length were detected under these conditions, and it was assumed, therefore, that further refining 


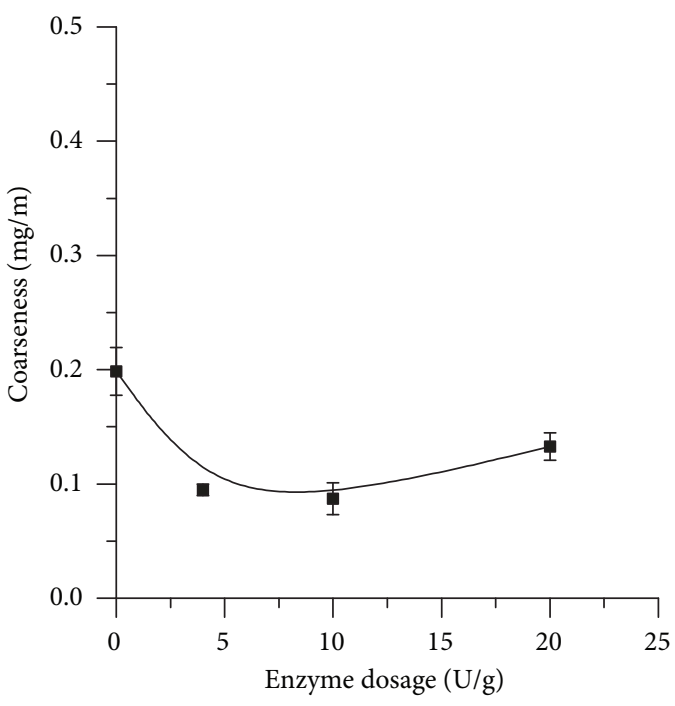

FIGURE 7: Effect of xylanase treatment on the coarseness of refiner mechanical fibers.

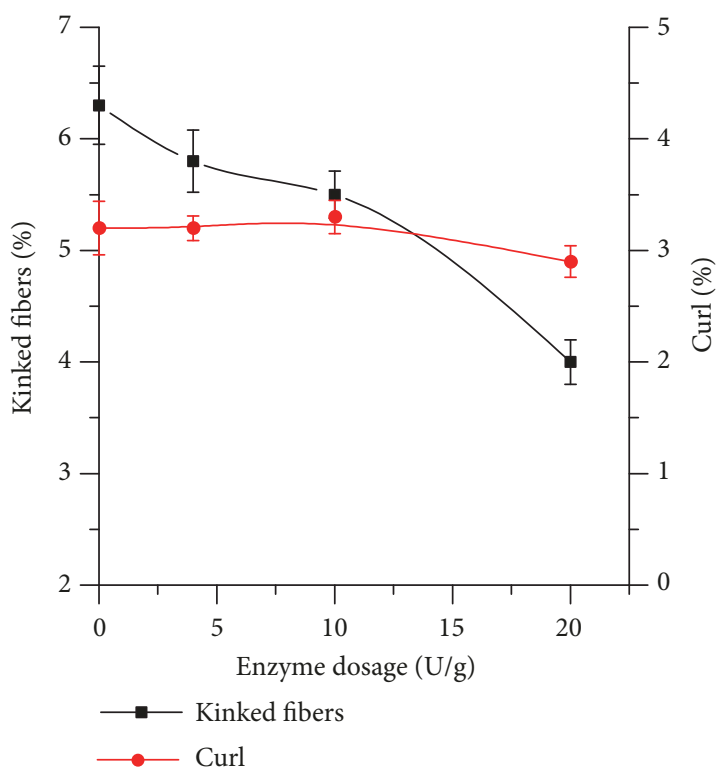

FIGURE 8: Effect of xylanase pretreatment on the fiber kinks and curls.

of the fiber bundles was taking place. With an increase in the enzyme dosage from 4 to $10 \mathrm{U} / \mathrm{g}$, no significant changes in the fiber coarseness are observed, although the fiber length decreases significantly (Figure 4), suggesting further fibrillation occurring during this refining process. Furthermore, an increase in the enzyme dosage from 10 to 20 $\mathrm{U} / \mathrm{g}$ results in a significant increase in the fiber coarseness, which is in agreement with the results presented in Figure 4 . However, this observation seemed counterintuitive. As shown in Figure 4, the fiber length and the pulp yield do not change significantly, whereas the fiber aspect ratio decreases. It was concluded, therefore, that the fibers flocculated at this stage because of more pronounced enzymolysis at the high xylanase dosages. However, no further relevant details are available, and the issue is currently under investigation.

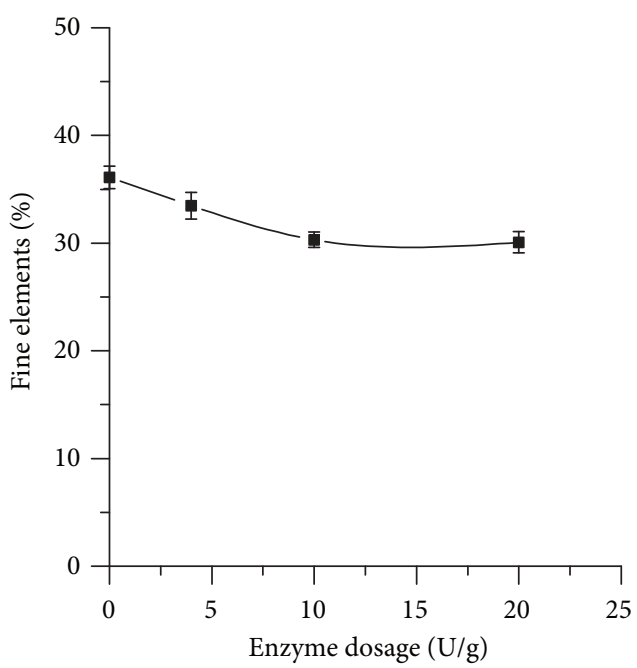

FIGURE 9: Effect of xylanase treatment on the fine element contents of the fibers.

3.3.3. Fiber Kinks and Curls. When fibers are subjected to stresses, such as shearing force, compressive force, friction, or distortion, deformation takes place to various degrees, which are characterized mainly as fiber curls and kinks [37]. Fiber curl refers to the gradual and continuous curvature of a fiber, whereas fiber kinks are abrupt bending changes in the fiber curvature. The observation of such fiber deformations suggests that the local ultrastructure of the fiber cell walls has been altered (i.e., in the secondary 2 layer $\left(S_{2}\right)$ in the fiber wall). During the pulping process at high concentrations, the heat and twisting arising from the gear grinding process result in the fibers being subjected to high thermal and mechanical stresses, which, in turn, lead to the formation of fiber kinks and curls. The effect of xylanase treatment on the kinks and curls present in the mechanically refined fibers is shown in Figure 8. More specifically, by increasing the enzyme dosage from 0 (the control sample) to $20 \mathrm{U} / \mathrm{g}$, the percentage of fiber curls decreases from 3.2 to $2.8 \%$, and the percentage of kinked fibers decreases from 6.3 to $3.8 \%$. Xylanase can diffuse through the fiber cell wall into the cell interior, thereby affecting both the fiber surface and the fiber interior [22]. Because of the degrading of hemicellulose to a certain degree, the fiber structure is loosened, which leads to improved water absorption and swelling capabilities of the fibers. Therefore, a higher enzyme dosage aids in reducing the appearance of curls and kinks in the fibers.

3.3.4. Fine Elements. A part of the fine components from the high-yield pulp was formed by the separation of the parenchyma cells and the vessel elements during pulping, while the other part, including fine fibers and microfibers, was detached from the fiber surface (length $<0.2 \mathrm{~mm}$, width $<5 \mu \mathrm{m})$ [38]. Figure 9 shows the effect of the xylanase treatment on the composition percentage of fine elements. It is clear that, at an enzyme dosage of $<10 \mathrm{U} / \mathrm{g}$, the fine element contents decrease significantly. However, a plateau is reached with enzyme dosages $>10 \mathrm{U} / \mathrm{g}$. These results suggested that 


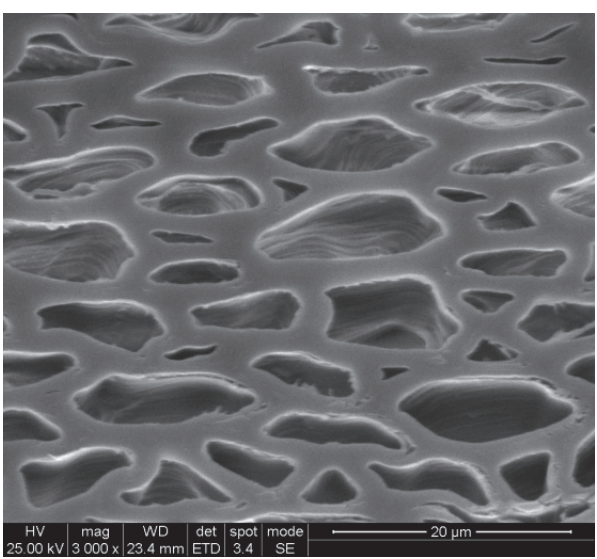

(a)

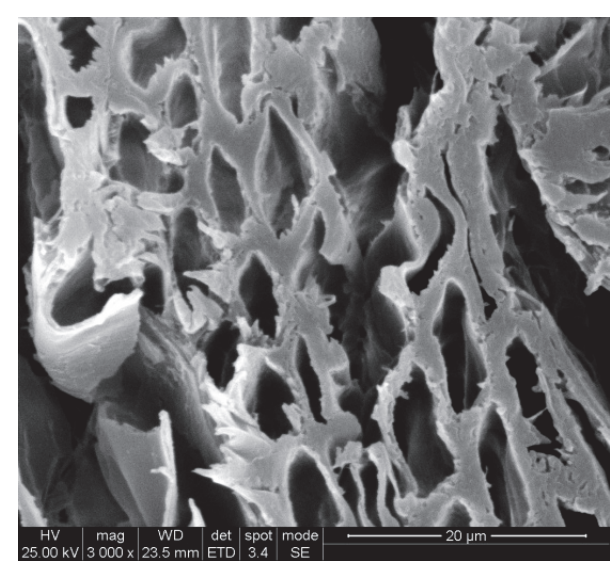

(b)

FIGURE 10: SEM images of the cross-sectional surfaces of mulberry branch fibers without and with xylanase pretreatment. (a) Control sample (without xylanase pretreatment). (b) Sample with xylanase pretreatment (enzyme dosage, $20 \mathrm{U} / \mathrm{g}$ ).

the xylanase treatment could reduce the cutting of fibers significantly.

\subsection{Effect of Xylanase Pretreatment on the Fiber Cross-} Sectional Morphology. SEM images of the cross-sectional surface of the materials with and without xylanase pretreatment after second-stage refining $\left(\mathrm{R}_{2}\right)$ are presented in Figure 10. As this figure indicates, when the xylanase treatment is not applied, the cross-section of the fibers shows a compacted structure without damage or cracks. However, with the xylanase treatment (Figure 10(b)), the fiber cross-section shows loosened structures, indicating that xylanase can degrade the xylan present in the mulberry branch and break the connections between xylan and lignin, thereby facilitating lignin dissolution. The result is that the fibers have loose structures, high porosity, and good swelling capability. After the xylanase treatment, the wood tissue becomes porous, which facilitates dissociation of the fibers, thereby reducing energy consumption. The xylanase treatment softened and loosened the fiber tissue, which could reduce the fiber cutting in the grinding process. Therefore, the treatment is conducive to increasing the proportion of medium and long fibers and improving the fiber quality. This finding is consistent with the results mentioned above. In addition, these observations are in agreement with those of previous studies [31].

\section{Conclusion}

In this study, the yield, energy consumption, fiber quality, and fiber cross-sectional changes during the mechanical refining of whole stems of mulberry branches with xylanase treatment were analyzed systematically. We found that the xylanase treatment could facilitate significant loosening of the tissues and fibers, thereby aiding defibration in the subsequent mechanical refining process. The xylanase treatment could save approximately $4 \%$ of energy at an enzyme dosage of $20 \mathrm{U} / \mathrm{g}$ in the subsequent mechanical refining process for producing fibers. The yield did change slightly and was maintained at $>85 \%$. The average fiber length was reduced after mechanical refining with the xylanase pretreatment, which was attributed to the treatment effectively decreasing the long fiber bundles. Furthermore, we found that the xylanase treatment had no effect on fiber curl, whereas the fiber kink ratio and the fine element content decreased. This method therefore represents a promising protocol for the efficient and environmentally friendly production of fibers using whole stem mulberry branches. In further study, in an effort to improve fiber quality, we will investigate the influence of different xylanase processing procedures on fiber properties.

\section{Data Availability}

The data used to support the findings of this study are included within the article.

\section{Conflicts of Interest}

The authors declare no conflicts of interest.

\section{Acknowledgments}

This research was funded by the National Natural Science Foundation of China (Grant no. 31070524) and the Major State Basic Research Development Program of China (Grant no. 2010CB732205).

\section{References}

[1] R. Kumar, S. Singh, and O. V. Singh, "Bioconversion of lignocellulosic biomass: biochemical and molecular perspectives," Journal of Industrial Microbiology and Biotechnology, vol. 35, no. 5, pp. 377-391, 2008.

[2] A. Zucaro, A. Fierro, and A. Forte, A Review on Potential Candidate Lignocellulosic Feedstocks for Bio-energy Supply Chain, Springer, Cham, Switzerland, 2019. 
[3] S. Ercisli and E. Orhan, "Chemical composition of white (Morus alba), red (Morus rubra) and black (Morus nigra) mulberry fruits," Food Chemistry, vol. 103, no. 4, pp. 1380-1384, 2007.

[4] X. He, J. Fang, Y. Ruan et al., "Structures, bioactivities and future prospective of polysaccharides from Morus alba (white mulberry): A review," Food Chemistry, vol. 245, pp. 899-910, 2018.

[5] Z. Guo, I. Yu-Xiu, L. Lei, and Y. Fang, "A comparative study on the morphology and basic density of raw fiber in mulberry,eucommia and recycled materials," Journal of Northwest Forestry University, vol. 32, no. 5, pp. 202-207, 2017.

[6] R. Prithivirajan, P. Balasundar, R. Shyamkumar et al., "Characterization of cellulosic fibers from Morus alba L. stem," Journal of Natural Fibers, vol. 16, no. 4, pp. 503-511, 2018.

[7] Q. K. Beg, M. Kapoor, L. Mahajan, and G. S. Hoondal, "Microbial xylanases and their industrial applications: a review," Applied Microbiology and Biotechnology, vol. 56, no. 3-4, pp. 326-338, 2001.

[8] V. Kumar, A. K. Dangi, and P. Shukla, "Engineering thermostable microbial xylanases toward its industrial applications," Molecular Biotechnology, vol. 60, no. 2, pp. 1-10, 2018.

[9] R. Gupta and Y. Y. Lee, "Mechanism of cellulase reaction on pure cellulosic substrates," Biotechnology and Bioengineering, vol. 102, no. 6, pp. 1570-1581, 2009.

[10] S. Nie, K. Zhang, X. Lin et al., "Enzymatic pretreatment for the improvement of dispersion and film properties of cellulose nanofibrils," Carbohydrate Polymers, vol. 181, pp. 1136-1142, 2018.

[11] S. Nie, C. Zhang, Q. Zhang et al., "Enzymatic and cold alkaline pretreatments of sugarcane bagasse pulp to produce cellulose nanofibrils using a mechanical method," Industrial Crops and Products, vol. 124, pp. 435-441, 2018.

[12] Y. Liu, M. Liu, H. Li, B. Li, and C. Zhang, "Characteristics of high yield pulp fibers by xylanase treatment," Cellulose, vol. 23, no. 5, pp. 1-9, 2016.

[13] O. Mertens, J. Gurr, A. Krause et al., "The utilization of thermomechanical pulp fibers in WPC: a review," Journal of Applied Polymer Science, vol. 134, no. 31, Article ID 45161, 2017.

[14] V. J. J. Prasad and P. Suresh Kumar, "A review of recent developments in natural fiber composites and their mechanical, thermal \& machinabilty properties," Advanced Materials Research, vol. 1148, pp. 61-71, 2018.

[15] G. Cave and P. Fatehi, "Adsorption optimization of a biomassbased fly ash for treating thermomechanical pulping (TMP) pressate using definitive screening design (DSD)," The Canadian Journal of Chemical Engineering, vol. 96, no. 8, pp. 16631673, 2018.

[16] J. Park, B. Jones, B. Koo et al., "Use of mechanical refining to improve the production of low-cost sugars from lignocellulosic biomass," Bioresource Technology, vol. 199, pp. 59-67, 2016.

[17] R. Gonzalez, H. Jameel, H.-M. Chang, T. Treasure, A. Pirraglia, and D. Saloni, "Thermo-mechanical pulping as a pretreatment for agricultural biomass for biochemical conversion," Bioresources, vol. 6, no. 2, pp. 1599-1614, 2011.

[18] P. Bajpai, Basic Overview of Pulp and Paper Manufacturing Process, Springer, Cham, Switzerland, 2015.

[19] M. J. Sabourin and P. W. Hart, "Enhanced fiber quality of black spruce (picea mariana) thermomechanical pulp fiber through selective enzyme application," Industrial \& Engineering Chemistry Research, vol. 49, no. 12, pp. 5945-5951, 2010.
[20] P. Bajpai, "Biotecnnology for pulp and paper processing," in Biopulping, Springer, Singapore, 2018.

[21] R. L. Giles, E. R. Galloway, G. D. Elliott, and M. W. Parrow, "Two-stage fungal biopulping for improved enzymatic hydrolysis of wood," Bioresource Technology, vol. 102, no. 17, pp. 80118016, 2011.

[22] S. Shuddhodana, M. N. Gupta, and V. S. Bisaria, "Stable cellulolytic enzymes and their application in hydrolysis of lignocellulosic biomass," Biotechnology Journal, vol. 13, no. 6, Article ID 1700633, 2018.

[23] X. Lin, Z. Wu, C. Zhang, S. Liu, and S. Nie, "Enzymatic pulping of lignocellulosic biomass," Industrial Crops \& Products, vol. 120, pp. 16-24, 2018.

[24] A. Walia, S. Guleria, P. Mehta, A. Chauhan, and J. Parkash, "Microbial xylanases and their industrial application in pulp and paper biobleaching: a review," 3 Biotech, vol. 7, p. 11, 2017.

[25] K. Zhang, Y. Zhang, D. Yan, C. Zhang, and S. Nie, "Enzymeassisted mechanical production of cellulose nanofibrils: thermal stability," Cellulose, vol. 25, no. 9, pp. 5049-5061, 2018.

[26] S. Grönqvist, T. K. Hakala, T. Kamppuri et al., "Fibre porosity development of dissolving pulp during mechanical and enzymatic processing," Cellulose, vol. 21, no. 5, pp. 3667-3676, 2014.

[27] J. J. Kaschuk, T. M. Lacerda, V. Coma, and E. Frollini, "Enzymatic hydrolysis of mercerized and unmercerized sisal pulp," Cellulose, vol. 24, no. 6, pp. 2437-2453, 2017.

[28] K. Wong, J. Richardson, and S. Mansfield, "Enzymatic treatment of mechanical pulp fibers for improving papermaking properties," Biotechnology Progress, vol. 16, no. 6, pp. 1025-1029, 2000.

[29] P. W. Hart, D. M. Waite, L. Thibault et al., "Selective enzyme impregnation of chips to reduce specific refining energy in alkaline peroxide mechanical pulping," Holzforschung, vol. 63, no. 4, pp. 418-423, 2009.

[30] X. Lei, Y. Zhao, K. Li, and A. Pelletier, "Improved surface properties of CTMP fibers with enzymatic pretreatment of wood chips prior to refining," Cellulose, vol. 19, no. 6, pp. 22052215, 2012.

[31] K. Buzała, P. Przybysz, J. Rosicka-Kaczmarek, and H. Kalinowska, "Comparison of digestibility of wood pulps produced by the sulfate and TMP methods and woodchips of various botanical origins and sizes," Cellulose, vol. 22, no. 4, pp. 27372747, 2015.

[32] A. Pelletier, K. Li, Y. Zhao, G. Court, J. Luo, and M. Frith, "Improvement of enzyme transport in wood chips for thermomechanical pulp refining," Carbohydrate Polymers, vol. 95, no. 1, pp. 25-31, 2013.

[33] P. Bajpai, Green Chemistry and Sustainability in Pulp and Paper Industry, Springer, Cham, Switzerland, 2015.

[34] E. Cannel, "Mechanical pulping technologies focus on reducing refning energy," Pulp \& Paper, vol. 73, no. 5, pp. 63-70, 1999.

[35] J. S. Tolan, "Enzymes, pulp and paper processing," in Encyclopedia of Bioprocess Technology, Wiley, New York, NY, USA, 2002.

[36] D. Fougere, K. Clarke, Y. Zhao, and K. Li, "Chemical-mechanical pretreatment of wood: reducing downsizing energy and increasing enzymatic digestibility," Biomass \& Bioenergy, vol. 80, pp. 17-29, 2015.

[37] D. H. S. Page, R. S. Jordan, and B. D. M. C. Barbe, "Curl, crimps, kinks and micro-compressions in pulp fibers-their origin, measurements and significance. Papermaking raw materials: their interaction with the production process and their effect on paper properties," in Proceedings of the Transactions of the 8th Fundamental Research Symposium, pp. 183-227, Oxford, Punton, London, UK, 1985. 
[38] N. H. Kamaludin, A. Ghazali, and R. W. D. Wan, "Characterisation of mechanical pulp fines from alkaline peroxide pulping of EFB," Iranian Red Crescent Medical Journal, vol. 1482, no. 10, pp. 296-301, 2012. 


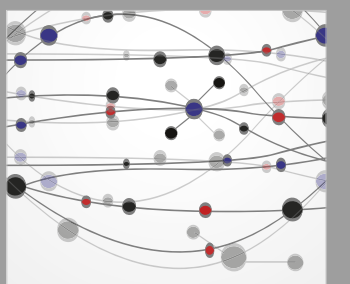

The Scientific World Journal
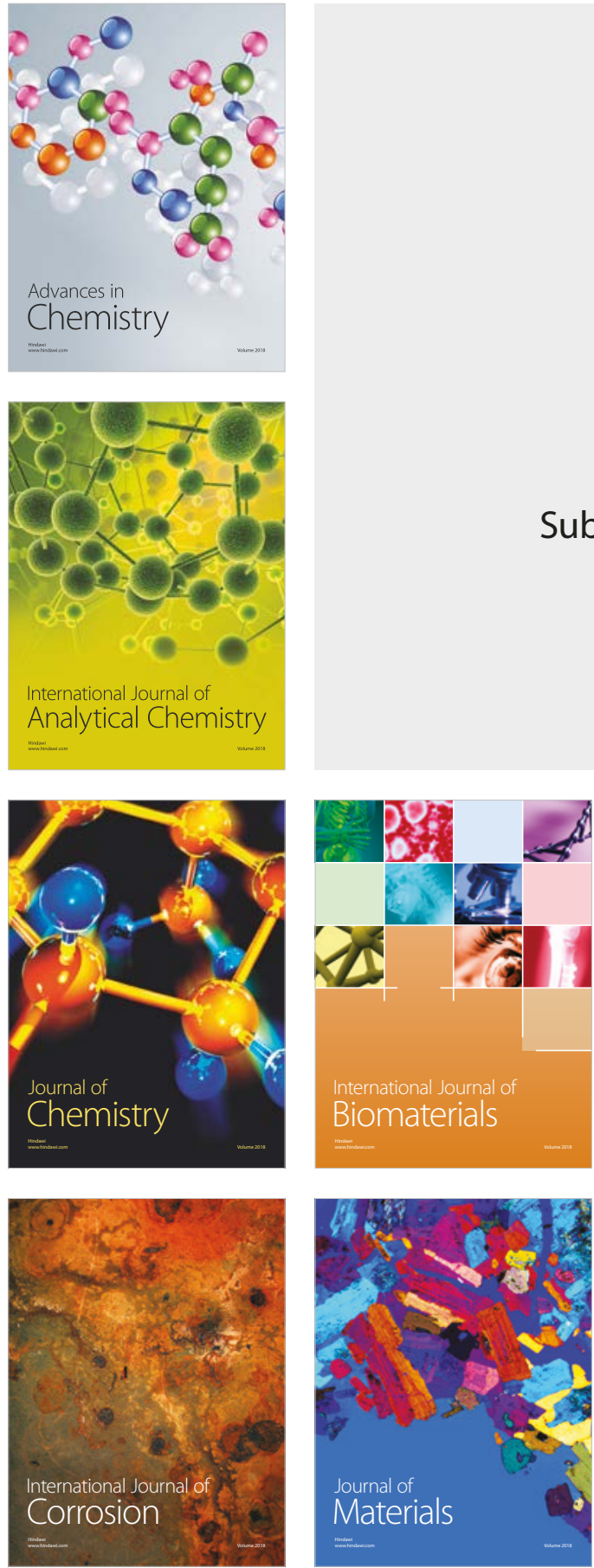

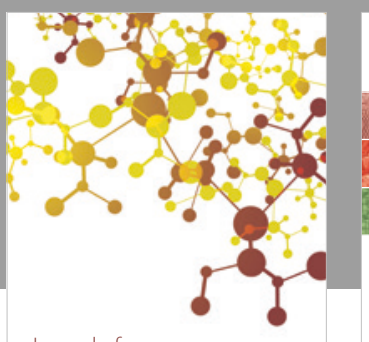

Journal of

Applied Chemistry
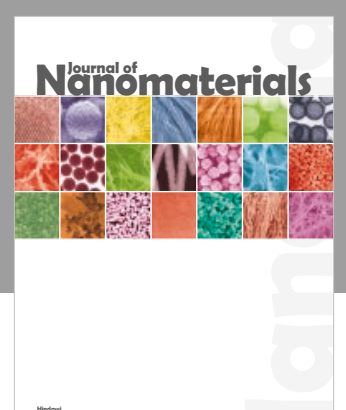

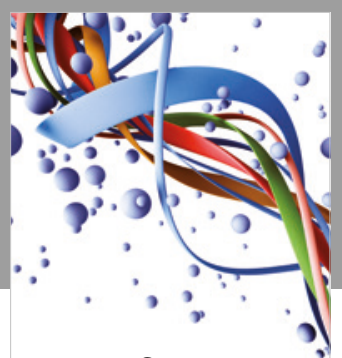

Scientifica

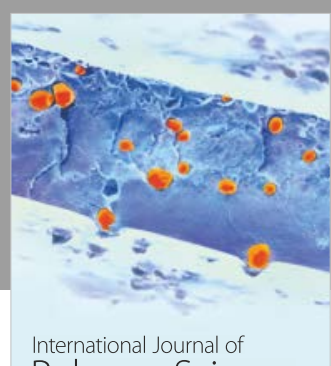

Polymer Science

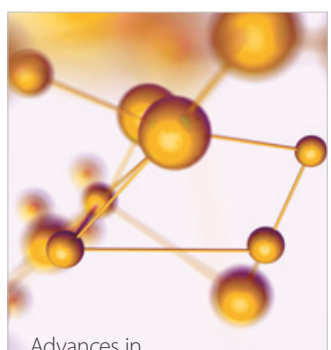

Physical Chemistry
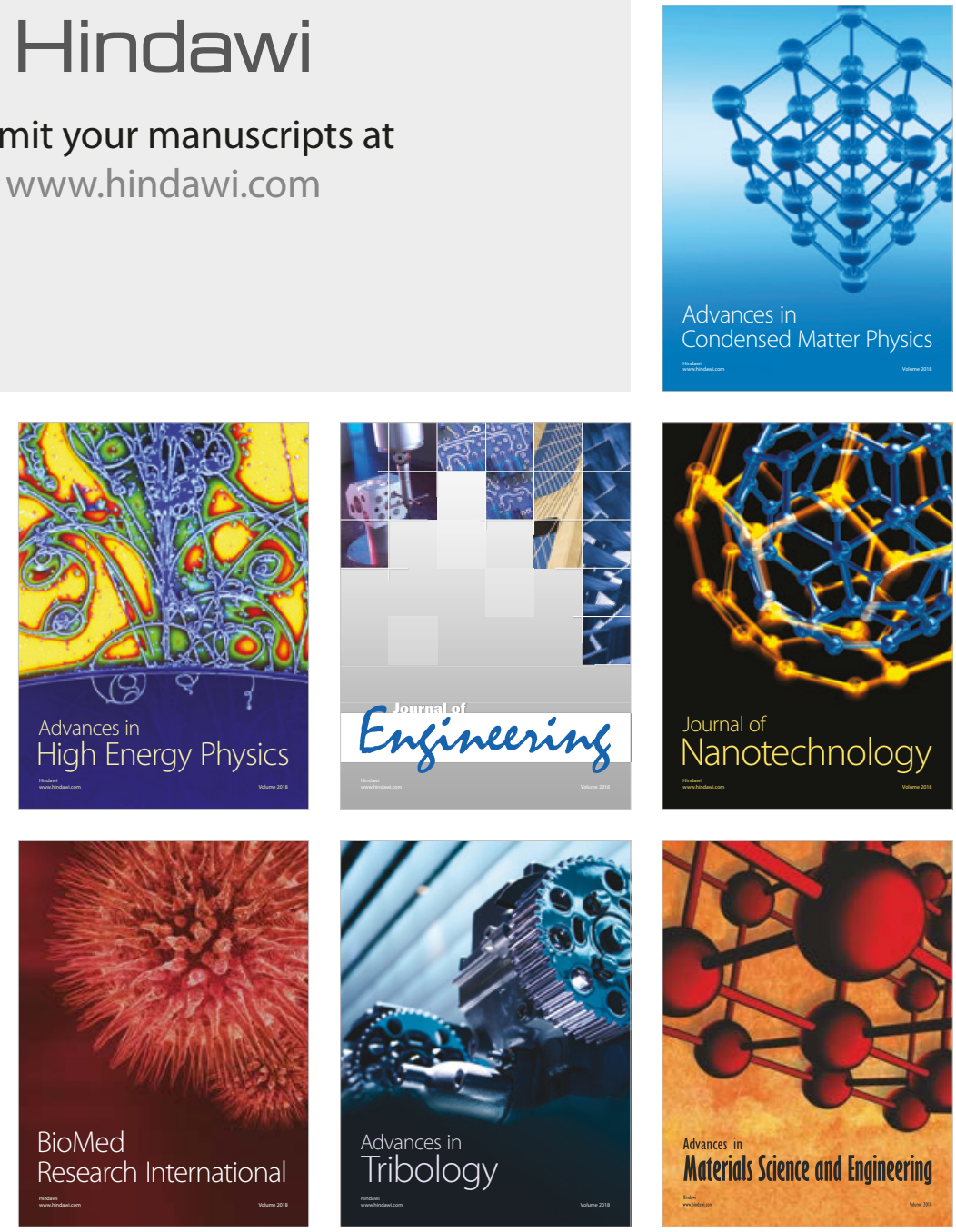